\title{
Prosthodontics 21: towards a new era?
}

\section{Asbjørn Jokstad}

\author{
Associate Professor, Institute of Clinical Dentistry, Dental Faculty, University of Oslo, \\ Norway
}

\begin{abstract}
As we enter a new century have we also entered a new era in prosthodontics?
Recent papers and editorials have started to address the issue of using an evidence-based approach in prosthodontics: are we now moving from an obsession with micro-measurement to one based on problem-solving?

Evidence-Based Dentistry (2002) 3, 2-4. DOI: 10.1038/sj/ebd/6400087
\end{abstract}

Has a new era in prosthodontics begun? Did it begin with the editorial published concurrently in the four leading international prosthodontic journals in 1994 entitled "Prosthodontics 21: a new beginning"? ${ }^{1}$ Did the statement made by GA Zarb in that editorial sum up the frustration many felt within the discipline?

"We have allowed ourselves to be perplexed in part by the ruthless demands of accuracy in our technical performances. We have also been obsessed with micro-measurements and the severe standards of a handicraft approach to problem solving."

To what extent this allegation is valid can be a matter for discussion. An observation, however, is that at the time Zarb's editorial was published, the number of evidence-based initiatives had expanded in the prosthodontic literature. The latest and most notable venture is the publication of the evidence-based dentistry series in the Journal of Prosthetic Dentistry earlier this year. ${ }^{2}$

One of the reasons why prosthodontics (indeed dentistry generally) has been slow to follow the EBD ethos is that randomised controlled trials, which lie at the heart of that ethos, are actually very difficult to conduct in many situations because of the heterogeneity of the starting point, the com- plexity of the outcome and, particularly, because of the relatively long time scale that most outcomes require.

\section{Environmental changes}

Why this gradually increasing focus on therapy-effectiveness outcomes in modern prosthodontics rather than technical performance? Several factors are perhaps responsible:

- An increasing number of elderly patients retain their teeth throughout life, often generating complex treatment decisions. Many papers report large discrepancies between professionally assessed need and subjective treatment demand, especially in the elderly. This led to, for instance, the formulation of the shortened-dental-arch concept in the mid-1980s, the subject of much debate within the prosthodontic community. At first it was regarded as clinical opinion only, but a large number of clinical studies, using an array of more or less appropriate study designs, have since been carried out to establish the theory's validity. $^{3}$

- The relationship between prosthodontics and oral physiology has always been very close, since it is related to the question of patient need versus demand. During the 1980 s an increasing number of papers questioned the many dogmas and statements on the association that were unsupported by science described in several traditional textbooks. Most notable among these is the etiological role of occlusal patterns of patients with temporomandibular disorders (TMD), culminating with the National Institutes of Health conference on TMD in $1996 .{ }^{4}$

- Tremendous advances have been made in the development of new implant biomaterials and techniques. In spite of very positive clinical results seen in several papers there is, however, resistance to using implants from traditionalists and prosthodontists remembering implantology from the pre-Brånemark era. Unfortunately, serious research on the benefits and potentials of implant based prosthodontics over traditional solutions has seemed to be delayed until fairly recently. The reason for this is debatable, but may be a combination of the manufacturers' race towards obtaining a share of the implant market and the indisputable fact that implantbased prostheses are preferred over traditional dentures by most patients. $^{5,6}$

- "Co-evolution" of technology in other disciplines such as orthodontics, periodontology and endodontics, as well as the introduction of new and complex oral surgical procedures, can be added to the potential of implant placements, dramatically broadening the reper- 
toire of oral rehabilitation. There is still a pressing need, however, to address efficacy versus effectiveness issues, i.e., whether the technical feasibility shown under often sophisticated high-tech circumstances can be realised under ordinary clinical circumstances in general practice.

- Manufacturers produce increasing numbers of new materials, instruments and dental equipment, which require revenue returns. As the information highway today reaches not only the dental professional but also individual patients and patient organisations the modern prosthodontists recognises a need to be adept in how to critically appraise new scientific knowledge.

The two editors of the International Journal of Prosthodontics, J Preston and GE Carlsson, reflected on many of the issues described above in some of their editorials. ${ }^{7-12}$ In so-doing, they raised the awareness of the prosthodontic community to these new challenges. An analogous question of how to best describe prosthodontics and what the aims of prosthodontic therapy should be, was a paper attempting to establish an appropriate definition of modern prosthetic dentistry. ${ }^{13}$

\section{Systematic reviews}

Meta-analysis of primary study data began in the medical literature towards the end of the 1980s. Meta-analyses of prosthodontic-therapy data were among the first in dentistry and were carried out by Creugers and van't Hof at the University of Nijmegen in The Netherlands. They reviewed durability data on resin-bonded bridges, ${ }^{14}$ post and core restorations, ${ }^{15}$ conventional fixed bridges, posterior resin-bonded bridges ${ }^{16}$ and, later, on anterior veneer restorations. ${ }^{17}$ Several other meta-analyses on prosthodontic topics have also appeared, e.g., on fixed partial dentures,${ }^{18}$ implants, ${ }^{19}$ implants in partial edentulism ${ }^{20}$ and implants placed in grafted maxillary sinuses. ${ }^{21}$ A systematic review on the relationship between the need for occlusal therapy and prosthodontic treatment has also recently been presented. ${ }^{22,23}$ Finally, a group at the University of Gainesville, Florida, USA, has searched the literature for randomised controlled trials in prosthodontic journals and presented their findings in two papers. ${ }^{24,25}$

\section{The future}

JD Anderson, from the University of Toronto in Canada, has for some time argued both in lectures and in the literature that there is a need for evidence-based practice in prosthodontics. ${ }^{26,27}$ Somebody has been listening: the first textbook in prosthodontics to include a chapter on evidence-based dentistry was published in $2000 .^{28}$ The topic has also begun to appear in meetings of prosthodontic societies. The annual meeting of the Scandinavian Society for Prosthetic Dentistry in 1998 had 'Evidence-based Care in Prosthetic Dentistry' as a main topic. ${ }^{29}$ Evidencebased dentistry was also a main topic at the annual meeting of the Swiss Society for Prosthetic Dentistry in 2000, and in 2001 at both the German Society for Prosthodontics and Dental Materials' and the Japanese Prosthodontic Association's annual meetings. The International College of Prosthodontists ICP - will focus on the issue at their next biannual meeting in Halifax, $\mathrm{Ca}$ nada in 2003, and an international meeting is planned to be held at the University of Toronto in the near future.

Finally, one of the most promising applications of evidence-based dentistry in prosthodontics is the systematic review that currently is being carried out within the Cochrane Collaboration on 'Interventions for replacing missing teeth with or without osseointegrated implants' ${ }^{30}$ The systematic review will be completed this year and thus form a basis for future developments of controlled clinical trials in prosthodontics.

\section{Evidence-based dentistry and treatment decisions}

It has always been acknowledged that all prosthodontic therapy includes high costs, an implicit biological price and a durability element. Thus, clinical decision-making was previously often a dichotomous decision whether prosthetic therapy should be carried out or not, or, at best, a choice between fixed, combined or removable prostheses. Today, the situation is that, first, there is a multitude of possible treatment modalities available; second, there are many complex patient scenarios because people do not lose teeth that show heavy signs of wear; and, third, there is an increasingly older population with varying degrees of disability associated with their health state. It is no wonder that many prosthodontists feel the need to learn how to critically appraise the levels of benefit versus harm of the various methods available, in order to be able to apply the appropriate modality, according to individual patients needs and preferences. This is evidencebased dentistry applied in practice.

1. Zarb GA. Prosthodontics 21: a new beginning. J Prosthet Dent 1994; 72:23A-24A.

2. McGivney GP. Evidence-based dentistry. J Prosthet Dent 2000; 83:11-12.

3. Luthardt $R$, Spieckermann J, Böning $K$, Walter $M$. Therapie der verkürzten Zahnreihe. Deutsch Zahnärztl Z 2000; 55:592-609.

4. National Institutes of Health. Management of temporomandibular disorders. Oral Surg Oral Med Oral Pathol Oral Radiol Endod 1997; 83:1-190.

5. Eckert SE, Parein A, Myshin HL, Padilla JL. Validation of dental implant systems through a review of literature supplied by system manufacturers. J Prosthet Dent 1997: 77:271-279.

6. Taylor TD, Agar J, Vogiatzi T. Implant prosthodontics: current perspective and future directions. Int J Oral Maxillofac Implant 2000; 15:66-75.

7. Preston JD. I don't know. Int J Prosthodont 1994; 7:199-200.

8. Preston JD. The information marketplace. Int J Prosthodont 1996; 9:215.

9. Preston JD. Taking advantage of the data deluge. Int J Prosthodont 1996; 9:509.

10. Carlsson GE. Opinion versus evidence. Int J Prosthodont 1997; 10:497.

11. Carlsson GE. Defining prosthodontics. Int J Prosthodont 1998; 11:293.

12. Carlsson GE. New directions in prosthodontics. Int J Prosthodont 1998; 11:537-538. 
13. Jokstad, A, Ørstavik J, Ramstad T. A definition of prosthetic dentistry. Int J Prosthodont, 1998; 11:295-301.

14. Creugers $\mathrm{NH}$, Van't Hof MA. An analysis of clinical studies on resin-bonded bridges. J Dent Res 1991; 70:146-149.

15. Creugers NH, Mentink AG, Kayser AF. An analysis of durability data on post and core restorations. J Dent 1993; 21:281-284.

16. Creugers $\mathrm{NH}$, Kayser $A F$, van't Hof MA. A meta-analysis of durability data on conventional fixed bridges. Community Dent Oral Epidemiol 1994; 22:448-452.

17. Kreulen $\mathrm{CM}$, Creugers $\mathrm{NH}$, Meijering AC. Meta-analysis of anterior veneer restorations in clinical studies. J Dent 1998; 26:345-353.

18. Scurria MS, Bader JD, Shugars DA. Metaanalysis of fixed partial denture survival: prostheses and abutments. J Prosthet Dent 1998; 79:459-464.

19. Esposito M, Hirsch JM, Lekholm U, Thomsen P. Biological factors contributing to failures of osseointegrated oral implants I. Success criteria and epidemiology. Eur J Oral Sci 1998; 106:527-551.

20. Lindh T, Gunne J, Tillberg A, Molin M. A meta-analysis of implants in partial edentulism. Clin Oral Implants Res 1998; 9:80-90.
21. Tong DC, Rioux K, Drangsholt $M$, Beirne OR. A review of survival rates for implants placed in grafted maxillary sinuses using meta-analysis. Int J Oral Maxillofac Implant 1998; 13:175-182.

22. De Boever JA, Carlsson GE, Klineberg IJ. Need for occlusal therapy and prosthodontic treatment in the management of temporomandibular disorders. Part I. Occlusal interferences and occlusal adjustment. J Oral Rehabil 2000a; 27:367-379.

23. De Boever JA, Carlsson GE, Klineberg IJ. Need for occlusal therapy and prosthodontic treatment in the management of temporomandibular disorders. Part II. Tooth loss and prosthodontic treatment. J Oral Rehabil 2000b; 27:647-659.

24. Dumbrigue HB, Esquivel JF, Jones JS. Assessment of MEDLINE search strategies for randomized controlled trials in prosthodontics. J Prosthodont 2000; 9:813.

25. Dumbrigue HB, Jones JS, Esquivel JF. Developing a register for randomized controlled trials in prosthodontics: results of a search from prosthodontic journals published in the United States. J Prosthet Dent 1999; 82:699-703.
26. Anderson JD. The need for criteria on reporting treatment outcomes. J Prosthet Dent 1998; 79:49-55.

27. Anderson JD. Need for evidence-based practice in prosthodontics. J Prosthet Dent 2000; 83:58-65.

28. Karlsson S, Nilner K, Dahl BL. A Textbook of Fixed Prosthodontics. The Scandinavian Approach. Stockholm: Förlagshuset Gothia, 2000; pp 337-356.

29. Scandinavian Society for Prosthetic Dentistry. Evidence Based Care in Prosthetic Dentistry. Annual Meeting. Oslo: Scandinavian Society for Prosthetic Dentistry, 1998. http://www.odont.uio.no/ prosthodont/oslo.htm.

30. Esposito M, Coulthard P, Worthington HV, Jokstad A, Thomsen P. Interventions for replacing missing teeth with or without osseointegrated implants (protocol for a Cochrane Review). Cochrane Library, Issue 4, 2000. Oxford: Update Software. 